\title{
Right Hemisphere Cerebral Infarction Due to Air Embolism from Percutaneous Lung Biopsy: A Case Report
}

\author{
Chaitanya Ahuja $^{1 *}$, Yama Kharoti ${ }^{1}$, Jeffery J. Critchfield ${ }^{2}$, Meghna Chadha ${ }^{2}$ \\ ${ }^{1}$ Dotter Institute, Oregon Health \& Science University, Portland, USA \\ ${ }^{2}$ Wayne State University, Detroit, USA \\ Email: ${ }^{*}$ drchaitanyaahuja@gmail.com
}

Received April 18, 2013; revised May 18, 2013; accepted May 25, 2013

Copyright (c) 2013 Chaitanya Ahuja et al. This is an open access article distributed under the Creative Commons Attribution License, which permits unrestricted use, distribution, and reproduction in any medium, provided the original work is properly cited.

\begin{abstract}
Percutaneous transthoracic needle biopsy is a common procedure in interventional radiology. Systemic arterial air embolism causing cerebral infarction is a rare but much feared complication of percutaneous lung biopsy. We present a comprehensive review of iatrogenic air embolism post-lung biopsy, a complication that is often sub optimally managed. Patho-physiology, clinical features, and risk factors and management are reviewed.
\end{abstract}

Keywords: Air Embolism; Lung Biopsy; Cerebral Infarction; Computed Tomography (CT) Guidance

\section{Introduction}

A CT-guided needle lung biopsy is a common intervenetional radiology procedure. Percutaneous lung biopsy is considered as a safe and effective method for obtaining a tissue diagnosis in patients with lung masses. Reported complications include pneumothorax, intrapulmonary hemorrhage, hemoptysis, air embolism, seeding of the biopsy tract, and death. The most common complication, pneumothorax, is easily treated by radiologists and is typically associated with no long-term sequela. Air embolism as a complication is extremely rare; however it can become fatal when it happens. Early diagnosis and rapid application of hyperbaric oxygen therapy are the mainstay of treatment.

\section{Case Summary}

An elderly gentleman with a known esophageal mass and multiple pulmonary nodules was referred for a CT guided FNA biopsy of a pulmonary nodule. The patient was placed in left lateral decubitus position on the CT table. A 19 gauge needle was advanced under CT fluoroscopy into a right pulmonary nodule and FNA samples were obtained in end inspiration (Figure 1). During the procedure the patient became verbally unresponsive and rapidly developed left sided hemiplegia. Subsequently, a partial seizure was witnessed on the CT table. The procedure was concluded and a non contrast head CT was

\footnotetext{
*Corresponding author.
}

obtained (Figure 2). The head CT showed gas within the right MCA (Middle Cerebral Artery) territory vessels. Hyperbaric oxygen therapy was initiated as per the institutional air embolism protocol. A subsequent brain MR showed T2/FLAIR hyperintensity in the right MCA territory along with diffusion restriction, compatible with cerebral infarction (Figure 3). The patient developed permanent disability as a result.

\section{Discussion}

Percutaneous lung biopsy is one of the most common procedures performed in radiology departments. It is the minimally invasive gold standard for the histopathologic investigation of lung masses. Compared with other percutaneous biopsy procedures, lung biopsy carries a higher risk of potential complications, including occasional reports of death. Very few case reports have been described in literature relating to cerebral infarction due to air embolism from percutaneous lung biopsy. The largest series of four cases has been reported from Korea [1].

Though rare, air embolization from percutaneous lung biopsy is a known and potentially devastating complication. Indeed, the few cases of death reported from this procedure are most often due to air embolism. It can cause subsequent myocardial infarction, intractable arrhythmia or stroke [2]. The primary mechanism for introduction of air embolism is the creation of an airway-pulmonary vessel fistula during the procedure [3]. Issues with technique, such as allowing the needle hub to 


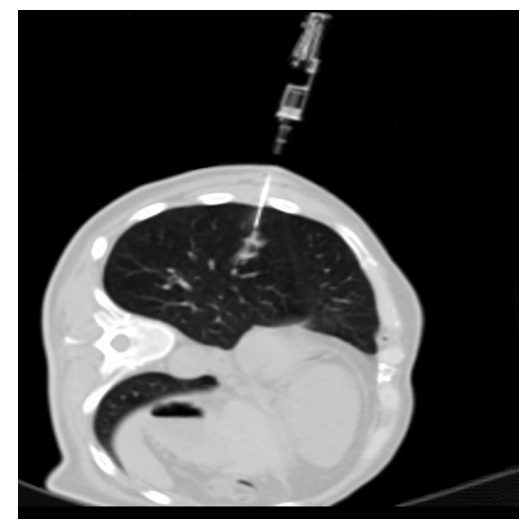

Figure 1. CT fluoroscopy image showing a percutaneous lung biopsy.

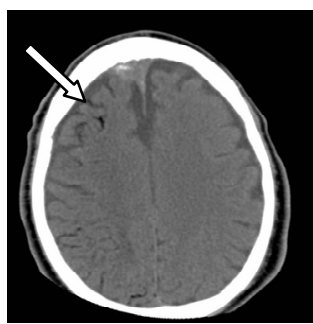

(a)

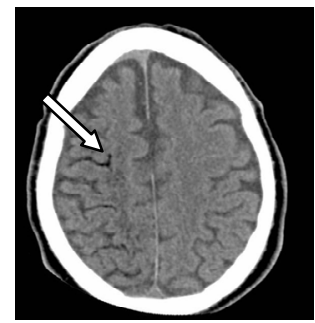

(b)

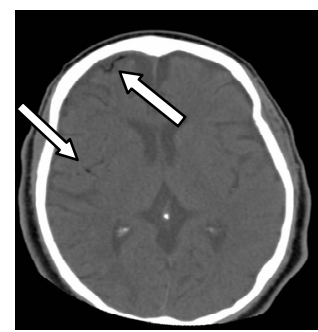

(c)

Figure 2. Non-contrast axial head CT images at three different levels showing serpiginous air within the right MCA territory distribution.

be open to outside air, can also result in air emboli.

There are three vessels that can receive air emboli during a lung biopsy. Air can enter a branch of the pulmonary arteries and lodge in the pulmonary capillary bed. While this can interfere with gas exchange and decrease pulmonary function locally, the lungs are well equipped to handle such situations via hypoxic vasoconstriction [4]. Air emboli in a bronchial artery will also lodge in the

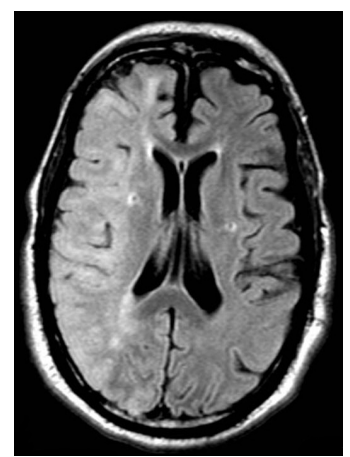

(a)

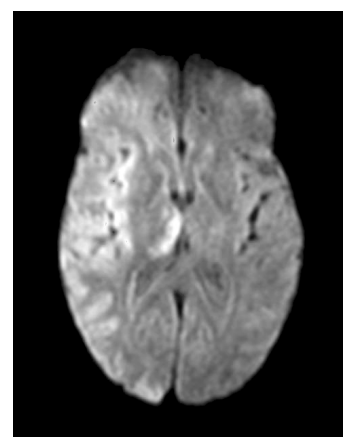

(b)

Figure 3. (a) Axial MR FLAIR image shows diffuse right middle cerebral artery territory edema. (b) Diffusion weighted image at the same level demonstrates increased diffusion restriction in the same region, compatible with cerebral infarction.

pulmonary capillary bed and have a similar net result. However, the more dreaded result is air entering a pulmonary vein, which flows into the left atrium and is therefore a conduit to the systemic circulation.

Once in the systemic circulation, an air embolus can lodge in any distal vessel. Emboli to either the coronary or cerebral arteries tend to result in the most profound morbidity [3,5]. In the case of the cerebral vasculature, there is a predilection for emboli to lodge in the right cerebral hemisphere [1]. Conventional aortic arch anatomy has the brachiocephalic artery as the first major branch of the aorta. It is also the largest of the three branches arising from the aortic arch. Further, the brachiocephalic artery is normally in a straight line with the trajectory of the ascending aorta. Air emboli therefore tend to select this vessel due to both its caliber and trajectory. Air then travels in a straight line through the right common carotid and right internal carotid arteries, ultimately lodging in the cerebral vasculature.

To prevent this catastrophic complication, multiple strategies for decreasing the incidence of air emboli have been discussed. Performing CT guided lung biopsies in suspended expiration, as opposed to end inspiration, will result in a higher intrathoracic pressure, which will decrease the likelihood of air emoblization. Higher gauge 
needles may also be used. In particular, FNA can be effectively done with a 21 or 22-gauge needle and will be less likely to induce air embolism than a 19-gauge or larger needle. Though diseased lung is often the target of a biopsy, the more diseased lung a needle is passed through, the greater the probability of inducing an air embolism is [6].

Vigilance for air embolism during lung biopsy can help decrease the most devastating complications, including stroke and death. Recognition of air within the left atrium, left ventricle or aorta is critical. Quickly placing the patient in Trendelenburg's position with their right side down will keep the air from embolizing distally. Interventional Radiology may then aspirate the air with an intravascular catheter. In the event of distal air embolism, hyperbaric oxygen therapy is the mainstay of therapy [7].

\section{REFERENCES}

[1] S. J. Um, S. K. Lee, D. K. Yang, C. Son, K. N. Kim, K. N. Lee and Y. S. Kim, "Four Cases of a Cerebral Air Embolism Complicating a Percutaneous Transthoracic Needle Biopsy,” Korean Journal of Radiology, Vol. 10, No. 1, 2009, pp. 81-84. doi:10.3348/kjr.2009.10.1.81
[2] A. R. Manhire, C. M. Richardson and F. V. Gleeson, "Lung Biopsy Guidelines-For the Obedience of Fools and Guidance of Wise Men,” Thorax, Vol. 58, No. 11, 2003, pp. 913-914. doi:10.1136/thorax.58.11.913

[3] R. A. Vanhulst, J. Klein and B. Lachmann, "Gas Embolism: Pathophysiology and Treatment," Clinical Physiology and Functional Imaging, Vol. 23, No. 5, 2003, pp. 237-246. doi:10.1046/j.1475-097X.2003.00505.X

[4] J. T. Sylvester, L. A. Shimoda, P. I. Aaronson and J. P. Ward, "Hypoxic Pulmonary Vasoconstriction," Physiological Reviews, Vol. 92, No. 1, 2012, pp. 367-520. doi:10.1152/physrev.00041.2010

[5] B. Mokhlesi, I. Ansaarie, M. Bader, M. Tareen and J. Boatman, "Coronary Artery Air Embolism Complicating a CT-Guided Transthoracic Needle Biopsy of the Lung," Chest, Vol. 121, No. 3, 2002, pp. 993-996. doi:10.1378/chest.121.3.993

[6] B. W. Arnold and W. J. Zwiebel, "Percutaneous Transthoracic Needle Biopsy Complicated by Air Embolism," American Journal of Roentgenology, Vol. 178, No. 6, 2002, pp. 1400-1402. doi:10.2214/ajr.178.6.1781400

[7] K. Ashizawa, H. Watanabe, H. Morooka and K. Hayashi, "Hyperbaric Oxygen Therapy for Air Embolism Complicating CT-Guided Needle Biopsy of the Lung,” American Journal of Roentgenology, Vol. 182, No. 6, 2004, pp. 1606-1607. doi:10.2214/ajr.182.6.1821606 